\title{
Rethinking cancer vaccines to avoid T-cell traps
}

\begin{abstract}
"While we can avoid the T-cell trap of incomplete Freund's adjuvant by the use of short-lived adjuvants, the only avoidance of the potential for a tumor T-cell trap is to vaccinate cancer patients earlier in their disease course."
\end{abstract}

\section{KEYWORDS: adjuvants - cancer vaccines - incomplete Freund's adjuvant - Montanide - T cells}

Cancer vaccines have gained increasing popularity since the US FDA approved the first cancer vaccine, sipuleucel-T, in 2010 and then approved the active specific immunotherapeutic agent, ipilimumab, in 2011. Several other recently reported positive randomized trials have started reversing the seemingly endless flow of failed cancer vaccine trials [1-4]. This has not been a complete reversal, however, with the recent report at ASCO that the Stimuvax trial failed to meet its primary end point in lung cancer; however, there was a significant improvement in overall survival in a large subset of over 800 patients. Since the majority of cancer vaccine trials have been performed in melanoma, this disease entity has endured more than its share of cancer vaccine failures. However, melanoma has taught us more than any other disease about the endogenous immune response to cancer. Specifically, multiple immunogenic peptides have been described from melanoma-specific antigens such as p-mel and MART-1 that efficiently induce and expand $\mathrm{T}$ cells with the capacity to recognize and destroy melanoma cells expressing these antigens. It is understandable then why these peptides have been the focus of many early and ongoing clinical trials. As with most vaccines, the antigenic focus of the vaccine must be joined with an immunoadjuvant to enhance its effectiveness. Incomplete Freund's adjuvant (IFA) is a water-in-oil emulsion specifically designed not only to enhance the immunogenicity of vaccines but also to serve as a slow-release source of antigen to the immune system. IFA has been one of the most commonly utilized cancer vaccine adjuvants to date and has been frequently used with peptide-based vaccines, especially in melanoma trials. Unfortunately, the vast majority of these peptide/IFA vaccine trials have been negative with rare objective clinical benefit demonstrated [5]. This has resulted in a variety of more complex vaccination strategies, the search for better tumor-associated antigens and the expansion of the cancer vaccine field beyond melanoma.

In a recently published study from the University of Texas, MD Anderson Cancer Center (USA), Hailemichael et al. have specifically addressed the clinical observation from multiple clinical trials that peptide/IFA cancer vaccines effectively and efficiently induce large numbers of peptide-specific cytotoxic $\mathrm{T}$ lymphocytes (CTLs), yet fail to produce measureable tumor responses [6]. In an elegant series of experiments, these authors systematically address how these peptide/IFA vaccines initially induce robust peptide-specific CTLs, yet ultimately lead to antigen depots that attract these antigen-specific T cells back to the sites of inoculation instead of to tumor sites. Once at the inoculation sites, these CTLs undergo antigen-driven dysfunction and ultimately deletion.

These authors used a short peptide gp100/IFA vaccine as a means to raise pmel- $1 \mathrm{~T}$ cells in a mouse model. After showing that this vaccine was effective in raising antigen-specific CTLs, they further show that these T cells did not persist and were unresponsive to boosting, unlike similar $\mathrm{T}$ cells that were induced with a virally delivered gp100 vaccine. Furthermore, the latter strategy was negatively impacted if the gp100/IFA vaccine was used to boost the pmel-1 T cells, resulting in a loss of these $\mathrm{T}$ cells and loss of substantial memory. These observations were confirmed with another antigen (ovalbumin)-based/IFA vaccine, suggesting that their findings were not restricted to gp100. Furthermore, they suggested that the mechanism of unresponsiveness was not the classical high-zone tolerance since their findings persisted even with small doses

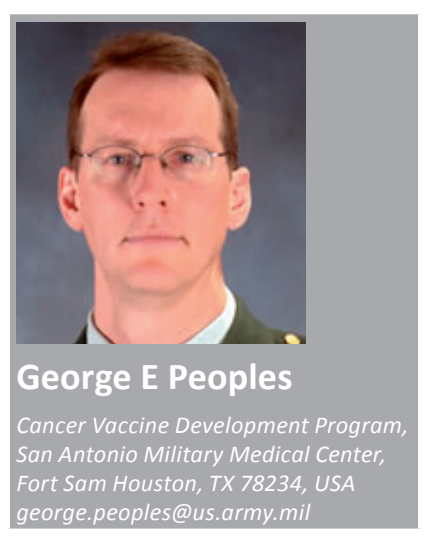

Future : $\because$ fS 
of peptide $(10 \mu \mathrm{g}) / \mathrm{IFA}$. Additionally, it did not appear that B cells or Tregs were involved in the induced hyporesponsiveness, since the latter persisted even in $\mathrm{B}$ cell-deficient $\mathrm{IgH}$ - and CD4-knockout mice.

The authors next tracked luciferase genetransduced pmel-1 CTLs transferred to vaccinated mice with gp100-expressing tumors. Mice vaccinated with the virally delivered gp100 vaccine resulted in the accumulation of pmel-1 $\mathrm{T}$ cells in the tumors, whereas animals vaccinated with gp100/IFA demonstrated large numbers of pmel-1 $\mathrm{T}$ cells at the site of vaccination and only slightly in the tumors. Additionally, the sequestration of these antigenspecific $T$ cells at the inoculation sites persisted for months. To confirm their findings, the authors also performed the experiments with clinical grade IFA, Montanide ISA-51 VG and human gp100 ${ }_{209-217(210 \mathrm{M})}$ in HLA-A2 transgenic mice, and found the same sequestration.

\section{"Several other recently reported positive randomized trials have started reversing the seemingly endless flow of failed cancer vaccine trials."}

Even more concerning than the diversion of these pmel-1 T cells away from the tumor was their ultimate fate once trapped at the vaccination site. The authors demonstrate that the prolonged exposure of pmel-1 T cells to antigen resulted in high rates of apoptosis, which required $\mathrm{T}$-cell receptor engagement, but T-cell deletion occurred regardless of T-cell receptor affinity and signaling strength. There also appeared to be an enhanced Fas expression on pmel-1 $\mathrm{T}$ cells exclusively at vaccination sites and $\mathrm{T}$-cell apoptosis seen there was somewhat abrogated in FasL knockout mice. Furthermore, IFN- $\gamma$, which is a known inducer of Fas, FasL and T-cell apoptosis, was found at sites of vaccination where it also promoted the accumulation of potent immunosuppressive myeloid-derived suppressor cells with upregulated FasL and PD-L1. The authors summarized these findings by stating "these data suggest that gp100/IFA vaccination induced antigendriven recruitment of the majority of the gp100-specific $T$ cells to the vaccine-draining lymph node and vaccination site, where they underwent priming and then antigen-driven apoptosis mediated by IFN- $\gamma$ and FasL in a T cell-hostile environment replete with myeloid cells expressing FasL and PD-L1.”
Interestingly, similar findings of IFAmediated sequestration and T-cell dysfunction did not apply to vaccines using longer peptides and IFA, which have shown some clinical success. The authors explain the latter by demonstrating that a longer gp100 peptide was presented by relatively rare dendritic cells in the vaccine-draining lymph node compared with the ubiquitous presentation of the short gp100 peptides; therefore, without the excessive antigen expression, there was no antigen-driven T-cell dysfunction and deletion. In an accompanying editorial to this article, those authors, after giving a nice history of oil emulsified vaccines, further expound on the potential benefits of the use of long peptides as a basis of vaccines (with or without IFA), which has been commonly advanced [7].

Hailemichael et al. further show that the sequestration, dysfunction and deletion associated with IFA can be attenuated to some extent by the addition of other immunostimulatory molecules such as the CD40 mAb, TLR7 agonist and IL-2, which they termed COVAX, to the gp100/IFA vaccine. In fact, the use of IL-2 as well as the incompleteness of the IFAmediated T-cell hyporesponsiveness is the authors' explanation for the positive Phase III trial of gp100/IFA peptide vaccination with systemic IL-2 versus IL-2 alone [1]. In the latter trial, the vaccination arm demonstrated both an improved progression-free survival as well as an improved overall survival. Therefore, in this instance, the systemic IL-2 was purportedly able to support the few gp100-specific T cells that escaped sequestration. The absence of IL-2 may also explain the poor results seen with this same vaccine in the control arm of the pivotal ipilimumab Phase III trial [8].

More importantly, the authors go on to show that compared with the gp100/IFA vaccine, a short-lived gp100 in saline and COVAX effectively raised pmel-1 $\mathrm{T}$ cells that efficiently localized to tumors. They proceed to demonstrate that the short-lived vaccine preparation prevented T-cell dysfunction and favored the early induction of effector T-cell phenotype that then transitioned to memory T-cell phenotype. While gp100/IFA-primed $T$ cells became effector memory type cells, the short-lived vaccine promoted the central memory phenotype. In summary, they state "a short-lived vaccine formulation induced longlived, functional $\mathrm{T}$ cells that localized preferentially to tumor sites, increasing antitumor activity." 
After discussing their findings in the context of multiple clinical trials using IFA with a variety of cancer antigens that have demonstrated reactivation of old inoculation sites, the authors finally conclude "the recognition that persistent antigen at vaccination sites induces strong sequestration and subsequent dysfunction and deletion of vaccination-induced $T$ cells directly points to desired characteristics of new classes of vaccines based on nonpersistent and rapidly biodegradable vaccine adjuvants." In addition to their suggestion for short-lived vaccine preparations, the authors clearly demonstrate the efficiency of short peptide-based vaccines for eliciting large numbers of tumor-reactive CTLs. Now we have at least some explanation for the ineffectiveness of these peptide-based vaccines using IFA in clinical trials despite the pronounced T-cell responses seen.

Our own research group's experience has been with peptide-based vaccines but using GM-CSF as an adjuvant as opposed to IFA and targeting HER2. The latter is a widely validated tumor-associated antigen in breast cancer that has been extensively studied, akin to gp100 in melanoma. Immunogenic short peptides have been described from the HER2 sequence and E75 is by far the most well-studied peptide [9]. E75 + adjuvant as a vaccine has been evaluated in a series of Phase I trials [10-13]. All of the trials have shown an effective and efficient expansion of E75-specific CTLs and all but one trial showed that these $\mathrm{T}$ cells had antitumor activity. Interestingly, the only trial to show the absence of antitumor killing was the only trial to use IFA [11]. The remainder of the trials utilized GM-CSF.

\section{“Our group's preferred approach to cancer vaccines is peptides (both short and long) mixed with GM-CSF and injected intradermally."}

GM-CSF as an adjuvant has also met with some criticism, but it is the immunoadjuvant in sipileucel-T, the only vaccine to reach FDA approval, as well as multiple other vaccines in late-phase testing. The issue of GM-CSF-induced myeloid-derived suppressor cells has only been reported in late-stage melanoma patients and appears to be at least partially dose-dependent. The benefits and potential issues of GM-CSF as an adjuvant have been thoroughly reviewed by our research group elsewhere [14].

Our group's preferred approach to cancer vaccines is peptides (both short and long) mixed with GM-CSF and injected intradermally. The skin is our largest immunologically active organ and is specially equipped for dealing with foreign or dangerous invaders. GM-CSF effectively induces a cellular infiltrate and helps mature professional APCs, which have ready access to the draining lymph node via the subdermal lymphatic plexus. Very little of the GM-CSF reaches the systemic circulation as it is rapidly consumed locally. As shown in the reviewed article, short peptides are efficiently and ubiquitously presented to the immune system. There has been a suggestion that this overpresentation of CTL epitopes may lead to tolerance (hence the potential benefit of longer peptides that require processing by professional APCs), yet this was not seen in the present study with the short-lived preparation vaccine. We have seen, in our Phase I/II trials of E75 + GM-CSF, very efficient expansion of peptide-specific $T$ cells with the ability to recognize and lyse HER2-expressing cells even at low levels of antigen expression [13,15-17]. Furthermore, these E75-specific CTLs can be repeatedly boosted over years without signs of unresponsiveness [18]. More importantly, these vaccine-specific CTLs appear to confer clinical benefit by reducing the recurrence rate in patients receiving the vaccine in the adjuvant setting. In fact, we have recently reported the final 5-year follow-up results of our E75 + GM-CSF Phase II trial in the adjuvant setting. Among the 187 patients (108 HLA-A2/A3 ${ }^{+}$vaccinated; 79 HLA-A2/A3 contols), the 60-month recurrence rate was reduced by $50 \%$ in the vaccinated patients [19].

The latter trial design separates our clinical studies from many other cancer vaccine trials that seek to treat established tumors in laterstaged patients. All of our trials have been aimed at clinically disease-free patients at risk of recurrence. Interestingly, we have been able to correlate the clinical benefit in our vaccinated patients with the magnitude of their immune response to the vaccine, a feat that has eluded most therapeutic vaccines [17]. However, the latter situation is complicated by the likely trafficking of $T$ cells to sites of disease burden. Unfortunately, the potential of T-cell trapping and antigen-driven T-cell dysfunction in a hostile environment may also apply to the site of an established tumor. While we can avoid the T-cell trap of IFA by the use of short-lived adjuvants, the only avoidance of the potential for a tumor T-cell trap is to vaccinate cancer patients earlier in their disease course. 


\section{Disclaimer}

The views expressed in this article are those of the author and do not reflect the official policy of the Department of the Army, Department of Defense or the US Government.

Financial \& competing interests disclosure

GE Peoples holds multiple patents on peptide vaccines to include E75. Many of these patents have been licensed to commercial entities. GE Peoples also consults for these entities in the further development and testing of these vaccines. The author has no other relevant affiliations or financial involvement with any organization or entity with a financial interest in or financial conflict with the subject matter or materials discussed in the manuscript apart from those disclosed.

No writing assistance was utilized in the production of this manuscript.

\section{References}

1 Schwartzentruber DJ, Lawson DH, Richards JM et al. gp100 peptide vaccine and interleukin-2 in patients with advanced melanoma. N. Engl. J. Med. 364, 2119-2127 (2011).

2 Schuster SJ, Neelapu SS, Gause BL et al. Vaccination with patient-specific tumorderived antigen in first remission improves disease-free survival in follicular lymphoma. J. Clin. Oncol. 29, 2787-2794 (2011).

3 Kantoff PW, Higano CS, Shore ND et al. Sipuleucel-T immunotherapy for castrationresistant prostate cancer. N. Engl. J. Med. 363, 411-422 (2010).

4 Kenter GG, Welters MJ, Valentijn AR et al. Vaccination against HPV-16 oncoproteins for vulvar intraepithelial neoplasia. $N$. Engl. J. Med. 361, 1838-1847 (2009).

5 Rosenberg SA, Yang JC, Restifo NP. Cancer Immunotherapy: moving beyond current vaccines. Nat. Med. 10, 909-915 (2004).

6 Hailemichael Y, Dai Z, Jaffarzad N et al. Persistent antigen at vaccination sites induces tumor-specific $\mathrm{CD} 8{ }^{+} \mathrm{T}$ cell sequestration, dysfunction, and deletion. Nat. Med. 19, 465-472 (2013).

7 Gnjatic S, Bhardwaj N. Antigen depots: T cell traps? Nat. Med. 19, 397-398 (2013).

8 Hodi FS, O’Day SJ, McDermott DF et al. Improved survival with ipilimumab in patients with metastatic melanoma. N. Engl. J. Med. 363, 711-723 (2010).

9 Mittendorf EA, Holmes JP, Ponniah S, Peoples GE. The E75 HER2/neu peptide vaccine. Cancer Immunol. Immunother. 57, 1511-1521 (2008).

10 Knutson KL, Schiffman K, Cheever MA, Disis ML. Immunization of cancer patients with a HER2/neu, HLA-A2 peptide, p369-377, results in short-lived peptidespecific immunity. Clin. Cancer. Res. 8, 1014-1018 (2002).

11 Zaks TZ, Rosenberg SA. Immunization with a peptide epitope (p369-377) from HER2/ neu leads to peptide-specific cytotoxic $T$ lymphocytes that fail to recognize HER $2 /$ neu $^{+}$ tumors. Cancer Res. 58, 4902-4908 (1998).

12 Murray JL, Gillogly ME, Przepiorka D et al. Toxicity, immunogenicity, and induction of E75-specific tumor-lytic CTLs by HER2 peptide E75 (369-377) combined with granulocytemacrophage colony-stimulating factor in HLA-A2 ${ }^{+}$patients with metastatic breast and ovarian cancer. Clin. Cancer Res. 8 , 3407-3418 (2002).

13 Peoples GE, Gurney JM, Hueman MT et al. Clinical trial results of a HER2/neu (E75) vaccine to prevent recurrence in high risk breast cancer patients. J. Clin. Oncol. 23, 7536-7545 (2005).

14 Clive KS, Tyler JA, Clifton GT et al. Use of GM-CSF as an adjuvant with cancer vaccines: beneficial or detrimental? Expert Rev. Vaccines 9, 519-525 (2010).

15 Peoples GE, Holmes JP, Hueman MT et al. Combined clinical trial results of a HER2/ neu (E75) vaccine for the prevention of recurrence in high risk breast cancer patients: US Military Cancer Institute clinical trials group study I-01 and I-02. Clin. Cancer Res. 14, 797-803 (2008).

16 Benavides LC, Gates JD, Carmichael MG et al. The impact of HER2/neu expression level on response to the E75 Vaccine: from US Military Cancer Institute Clinical Trials Group study I-01 and I-02. Clin. Cancer Res. 15, 2895-2904 (2009).

17 Mittendorf EA, Clifton GT, Holmes JP et al. Clinical trial results of the HER-2/neu (E75) vaccine to prevent breast cancer recurrence in high-risk patients: from US Military Cancer Institute Clinical Trials Group study I-01 and I-02. Cancer 118, 2594-2602 (2012).

18 Holmes JP, Clifton GT, Patil R et al. Use of booster inoculations to sustain the clinical effect of an adjuvant breast cancer vaccine: from US Military Cancer Institute Clinical Trials Group study I-01 and I-02. Cancer 117, 463-471 (2011).

19 Vreeland TJ, Clifton GT, Hale DF et al. Final results of the Phase I/II trials of the E75 adjuvant breast cancer vaccine. Cancer Res. 72(Suppl. 24), P5-16-02 (2012). 